\title{
Treatment challenges in the management of moderate-to-severe plaque psoriasis - role of secukinumab
}

This article was published in the following Dove Press journal:

Clinical, Cosmetic and Investigational Dermatology

II October 2016

Number of times this article has been viewed

\author{
Mona Malakouti \\ Sharon E Jacob \\ Nancy J Anderson \\ Department of Dermatology, Loma \\ Linda University, Loma Linda, CA, USA
}

\begin{abstract}
Psoriasis is a chronic inflammatory skin disease that has a negative impact on psychosocial well-being and cardiometabolic health. Treatment options for moderate-to-severe psoriasis have expanded with the development of interleukin-17 (IL-17) inhibitors, the first of which is now available - secukinumab. Secukinumab is a fully human monoclonal immunoglobulin G1 $\kappa$ antibody that selectively inhibits the ligand IL-17A. In head-to-head studies, it is more effective than etanercept and ustekinumab, particularly in achieving Psoriasis Area and Severity Index (PASI) 90/100 and achieving PASI 50/75 as early as week 4. No head-to-head trials are available for comparison of adalimumab to secukinumab. Significant improvement in health care-related quality of life was also observed using the dermatology quality index in clinical studies. Safety data for secukinumab is comparable to available biologics. Specific safety concerns for the use of secukinumab include its use in patients with inflammatory bowel disease, reversible transient neutropenia, in those with a latex allergy, and the occurrence of mild to moderate oral or genital candidiasis. Secukinumab is an effective and safe treatment option that achieves high clearance rates up to PASI 90 and 100 as monotherapy in cases of moderate-to-severe psoriasis. It may be particularly helpful in patients with psoriasis who have formed antidrug antibodies or failed other biologic agents and in patients with psoriatic arthritis or ankylosing spondylitis.
\end{abstract}

Keywords: psoriasis, biologics, secukinumab, inflammation, quality of life

\section{Introduction}

Psoriasis is a common chronic inflammatory skin disease that occurs in $\sim 3 \%$ of the US general population. ${ }^{1}$ The impact of psoriasis on patients' lives can be devastating, as patients suffer from social stigmatization, low self-esteem, high medical costs in the setting of reduced work productivity, and ultimately a decreased quality of life (QOL)., ${ }^{2,3}$ Moderate-to-severe psoriasis has also been associated with multiple comorbidities including psoriatic arthritis, and cardiometabolic diseases such as obesity, hypertension, dyslipidemia, and diabetes mellitus., ${ }^{4,5}$

The treatment of moderate-to-severe psoriasis, or skin involving more than $10 \%$ body surface area ( $1 \%$ body surface area is equivalent to one whole hand), requires systemic agents. ${ }^{6}$ Previously, these patients were restricted to oral agents such as cyclosporine, methotrexate, and acitretin; the use of these agents is limited by generalized immune suppression and kidney and liver toxicity. ${ }^{6}$ Although phototherapy remains an efficacious treatment option, its use has been limited by cost, access, and inconvenience; the use of home ultraviolet therapy decreases these obstacles, yet remains
Correspondence: Sharon E Jacob

Department of Dermatology, Loma Linda University, II 370 Anderson Street, Suite 2600, Loma Linda, CA 92354, USA

$\mathrm{Tel}+\mathrm{I} 9095582890$

Fax + I 9095582448

Emailsjacob@contactderm.net 
underutilized. The treatment options for moderate-to-severe psoriasis have expanded the production of biologic agents designed to inhibit specific immune cell targets thought to play a role in the pathophysiology of psoriasis.

Initially thought to be a T-helper subset 1 (Th1)-mediated disease, tumor necrosis factor-alpha (TNF $\alpha$ ) neutralization/ blockade by biologic agents etanercept, infliximab, and adalimumab was pursued for the treatment of psoriasis and psoriatic arthritis. ${ }^{7,8}$ Ustekinumab, which blocks interleukin-12 (IL-12) and IL-23 by the common p40 subunit, provided an important link between IL-23 and the discovery of the downstream T-helper subset 17 (Th17) and IL-17 pathway. ${ }^{9-11}$ IL-23 acts to maintain and promote the growth of Th17 cells responsible for the release of IL-17A, a potent member of the IL-17 family of cytokines that plays a significant role in the pathogenesis of psoriasis. ${ }^{11}$ This discovery has led to the production of targeted biologic agents that directly inhibit these downstream products more proximal to the keratinocyte. The first US Food and Drug Administration (FDA) approved anti-IL-17 biologic is secukinumab. Other IL-17 inhibitors, such as brodalumab and ixekizumab, are undergoing further Phase III clinical trials. ${ }^{12,13}$ This review will discuss the role and utility of secukinumab in clinical practice.

\section{Methods}

We reviewed the results of published Phase II and Phase III clinical trials for secukinumab. For additional resources, we conducted an English literature search with search terms "secukinumab" and "psoriasis" using PubMed to locate additional research articles reviewing secukinumab in other disease states as well as psoriasis. We also reviewed in-text citations within these resources to identify other additional articles. Articles were screened for relevance by reviewing the title and abstract.

\section{Review of pharmacology, mode of action, and pharmacokinetics of secukinumab}

Secukinumab (Cosentyx ${ }^{\text {тм}}$; Novartis Pharma AG, Basel, Switzerland) is a fully human monoclonal immunoglobulin G1 $\kappa$ antibody that selectively inhibits the ligand IL-17A and its downstream effects by preventing it from binding to the IL-17 receptor. ${ }^{14}$ This inhibition prevents the activation of keratinocyte proliferation, release of further inflammatory cytokines, neutrophil activation, and angiogenesis. ${ }^{11}$

The bioavailability of secukinumab was $\sim 77 \%$, with peak concentrations being achieved 5-6 days after a single subcutaneous dose (150 or $300 \mathrm{mg}$ ). With monthly dosing as recommended, steady state is achieved within 20 weeks. The volume of distribution is 7.1-8.6 L for a single intravenous dose, representing the limited distribution to peripheral compartments.Microperfusion studies of the dermis illustrated the distribution of secukinumab to dermal interstitial fluid. ${ }^{15}$ Similar to the metabolism of endogenous immunoglobulins, secukinumab is metabolized intracellularly into small peptides and amino acids with a half-life elimination of $\sim 27$ days. Due to intracellular catabolism, the potential for drug interactions with secukinumab is low.

No dose adjustments for geriatric populations or those with renal or hepatic impairment are recommended. ${ }^{16}$ Patients are still able to mount a protective antibody response to inactivated or nonlive vaccinations while actively undergoing therapy with secukinumab. ${ }^{17}$

Typically, total serum IL-17A level (free IL-17A plus IL-17A complexed with secukinumab) rises after administration of secukinumab and declines near the end of the treatment period due to slow clearance of the IL-17A/ secukinumab complex. The clinical improvement of psoriatic lesions was paired with immunohistochemical studies of lesional biopsies. At baseline, lesional biopsies from psoriatic plaques showed higher levels of IL-17A compared to lesional biopsies from healthy volunteers. After treatment and improvement in the Psoriasis Area and Severity Index (PASI), lesional biopsies from psoriatic plaques showed decreased keratinocyte parakeratosis and thickness as well as decreased numbers of innate immune cells and CD3+ T-cells.

\section{Secukinumab dosing and efficacy}

To establish the dosing, efficacy, and safety of secukinumab, four Phase II and six Phase III trials were completed. Relevant Phase III trial results are given in Table 1.

Based on the dose-ranging, regimen-finding studies and the Phase III trials, the recommended dosing of secukinumab is $300 \mathrm{mg}$ subcutaneous injections (two $150 \mathrm{mg}$ injections) given at weeks $0,1,2,3$, and 4 followed by monthly maintenance dosing. A Phase II trial established the ability of secukinumab to achieve PASI 75 at week 12 in $57 \%$ of patients receiving $75 \mathrm{mg}$ and $82 \%$ of patient receiving $150 \mathrm{mg}$ compared to $9 \%$ of patients in the placebo group $(P<0.0001$ and $P<0.0002$, respectively). ${ }^{18}$ The $150 \mathrm{mg}$ dose was most efficacious if loaded with weekly dosing for the first month followed by monthly maintenance dosing. ${ }^{18,19}$ The Phase III trials that followed tested loading doses of 150 and $300 \mathrm{mg}$ for the first month and established that maintenance of PASI with fixed monthly maintenance dosing was greater than retreating patients upon clinical relapse in the Sculpture trial. ${ }^{20}$ 
Table I Secukinumab Phase III trials efficacy and safety

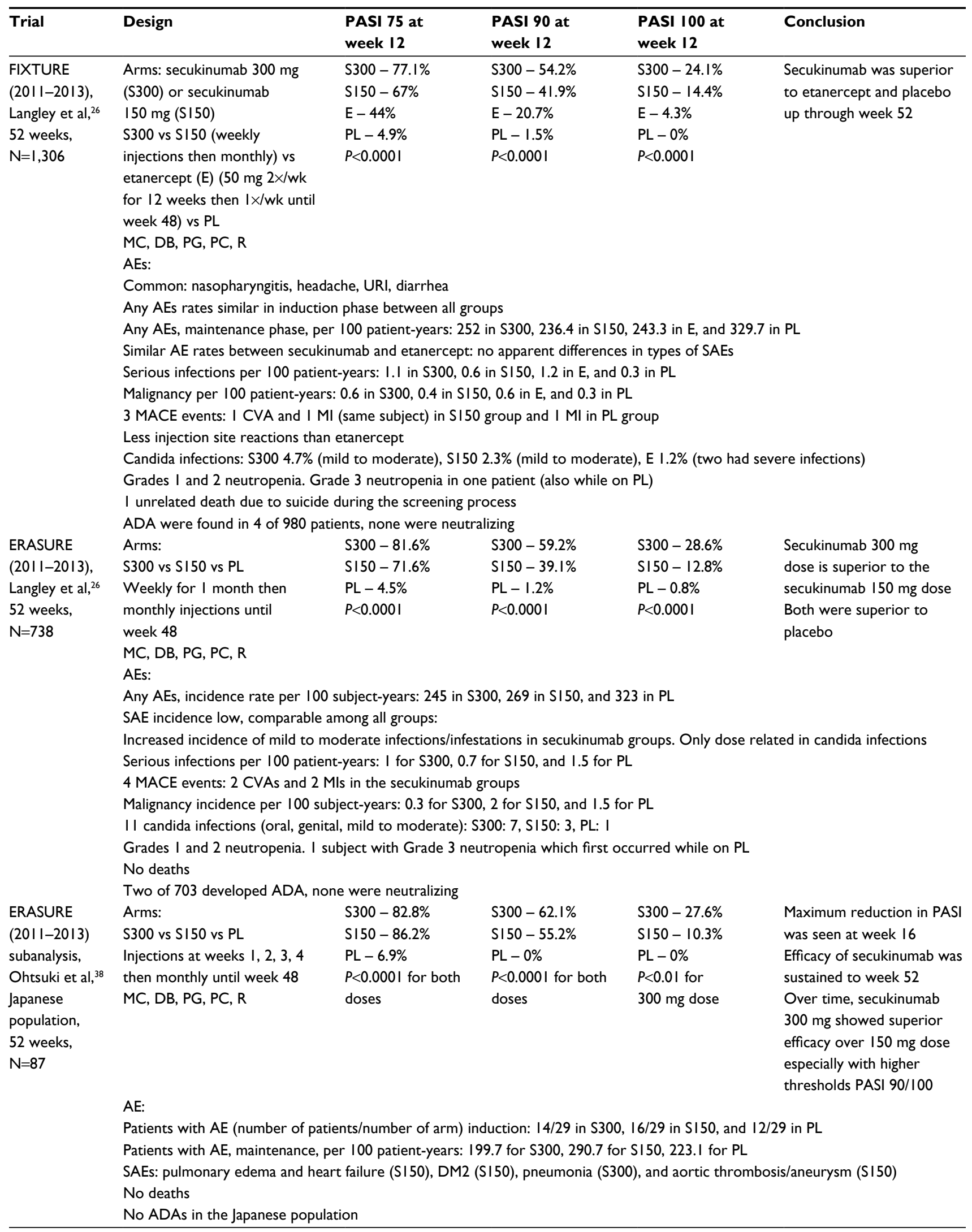


Table I (Continued)

\begin{tabular}{|c|c|c|c|c|c|}
\hline Trial & Design & $\begin{array}{l}\text { PASI } 75 \text { at } \\
\text { week I } 2\end{array}$ & $\begin{array}{l}\text { PASI } 90 \text { at } \\
\text { week I } 2\end{array}$ & $\begin{array}{l}\text { PASI } 100 \text { at } \\
\text { week } 12\end{array}$ & Conclusion \\
\hline JUNCTURE & Self-injection with & S300-86.7\% & S300 - 55\% & $S 300-26.7 \%$ & The autoinjector makes it \\
\hline$(20|2-20| 3)$ & autoinjector/pen: & SI $50-71.7 \%$ & $S I 50-40 \%$ & $S 150-16.7 \%$ & easy for patients to safely \\
\hline Paul et al, ${ }^{23}$ & S300 vs SI 50 vs PL & $P L-3.3 \%$ & $P L-0 \%$ & $P L-0 \%$ & and confidently self inject. \\
\hline $\begin{array}{l}12 \text { weeks, } \\
N=182\end{array}$ & MC, DB, PG, R & $P<0.0001$ & $P<0.000 I$ & $P<0.0006$ & $\begin{array}{l}\text { The autoinjector needle } \\
\text { is hidden and is activated } \\
\text { by simply placing the cap } \\
\text { against the skin }\end{array}$ \\
\hline
\end{tabular}

Any $A E$, number of patients/number of arm: $42 / 60$ in S300, 39/6I in SI50, and 33/6I in PL

SAE: I in S300, 2 in SI50, and I in PL

A case of serious pneumonia and malignant melanoma in situ reported unrelated to study drug

No MACE

I vulvovaginal and I oral candidiasis, mild infections

Neutropenia in $5 \%$ of S300, 8.2\% of SI50, and 3.3\% in PL, Grade I or 2

No deaths

No ADA antibodies

\section{SCULPTURE S300 and SI50}

(20II-20I3),

Mrowietz

et $\mathrm{al}^{20}$

52 weeks,

$\mathrm{N}=966$,

multicenter,

double-blinded

parallel group
Fixed regimen (every 4 weeks,

$\mathrm{FR}$ ) vs retreat as needed (RN)

(received treatment to week

12 followed by PL until loss of

$20 \%$ of maximum PASI score

or loss of PASI 75 response)

MC, DB, PG, R
PASI 75 at week 12:

S300-90.1\%

SI 50-84.4\%

Maintenance of PASI 75 at Week 40/52:

S300 RN - 67.7\%; SI50 RN - 52.4\%

S300 FR - 78.2\%; SI50 FR - 62.1\%

Mantel-Haenszel risk difference with 98.75 confidence used to determine noninferiority analysis

$A E$ rates were similar between all treatment arms (no placebo arm in this trial)

Most common AEs: nasopharygnitis, headache, URI

SAE rates were similar in induction phase

SAE rates in maintenance phase: I4 S300 RN, I8 S300 FR, I3 SI50 RN, I 2 SI50 FR

Two MACE events - acute $\mathrm{MI}$ and fatal cerebral hemorrhagic stroke (study investigator: unrelated)

One death due to CVA

Six total neoplasms

I 3 candida infections, all mild to moderate oral or genital candidiasis

Grade 3 neutropenia in 7 patients, transient no serious infections association

About four of 966 patients had ADAs. Two were neutralizing but not associated with decreased efficacy or pharmacokinetic abnormalities

\section{FEATURE}

(2012-2013),

Blauvelt et al, ${ }^{24}$

12 weeks,

$\mathrm{N}=177$,

double-blinded

parallel group multicenter,

\section{Evaluation of self-injection with}

prefilled syringes (PFS)

S300 vs SI 50 vs PL PFS

MC, DB, PG, PC, R

AEs:

Any AE, similar among groups (number of patients/number of arm): 30/59 in S300, 34/59 in SI50, and 28/59 in PL

SAEs: 3 in S300, 0 in SI50, and I in PL, sciatica and exfoliative dermatitis + 2 MACE

Severe infections: 3 cases, I:I:I in S300, SI50, and PL

Two MACE events: CVA and $\mathrm{MI}$ in patients with multiple cardiac risk factors prior to starting the study No deaths

Three cases of mild to moderate vulvovaginal candidiasis in S300 and SI50

Grade I neutropenia in 5\%-9\% of patients, I patient with Grade 2 neutropenia. None of these patients had serious infections

CLEAR

(20|4),

Thaci et al, ${ }^{25}$

52 weeks,

$\mathrm{N}=676$

\begin{tabular}{|c|c|c|c|c|}
\hline S300 mg vs ustekinumab & Week I2: & Week I2: & Week I2: & Secukinumab is superior to \\
\hline (dosing per protocol) & $S 300-91$ & S300 - 72.8 & S300 - 38.9 & ustekinumab in efficacy and \\
\hline \multirow[t]{6}{*}{$\mathrm{MC}, \mathrm{DB}, \mathrm{PG}, \mathrm{PC}, \mathrm{R}$} & Ustekinumab - 79.I & Ustekinumab - 53.4 & Ustekinumab - 25.7 & comparable in safety \\
\hline & $P=0.0003$ & $P<0.0001 \%$ & $P<0.0001 \%$ & \\
\hline & Week 16: & Week I6: & Week I6: & \\
\hline & S300 - 93.1 & S300 - 79 & $S 300-44.3$ & \\
\hline & Ustekinumab - 82. I & Ustekinumab - 57.6 & Ustekinumab - 28.4 & \\
\hline & $P=0.0001 \%$ & $P<0.0001 \%$ & $P<0.0001 \%$ & \\
\hline
\end{tabular}

Regular 4 weeks dosing of secukinumab or the fixed regimen is more efficacious for maintenance therapy 


\begin{tabular}{lllll}
\hline Trial Design & PASI 75 at & PASI 90 at & PASI I00 at & Conclusion \\
& week 12 & week 12 & week 12 & \\
\hline
\end{tabular}

AEs:

Any AE, similar between S300 (62\%) vs ustekinumab (58\%) (no placebo arm)

Mild to moderate infections were most common $29 \%$ S 300 and $25 \%$ ustekinumab.

SAEs 10 in S300 and 10 in ustekinumab groups

Three MACE: I unstable angina, I Ml, and I embolic stroke (dosing groups/drug not listed)

One malignancy: lung adenocarcinoma

One injection site reaction - nausea/vomiting

Two hepatic: transaminitis while on INH

Three serious infections: appendicitis, diverticulitis, and scrotal abscess

No deaths

Candida infections: twelve cases mild, oral, or genital mucocutaneous infections, localized

Ten cases of oral herpes

No neutropenia, IBD, or tuberculosis

Abbreviations: MC, multicenter; R, randomized; DB, double-blinded; PG, parallel grouped; PC, placebo-controlled; AE, adverse events; PL, placebo; SAE, serious adverse events; MACE, major adverse cardiac events; CVA, cerebrovascular accident; MI, myocardial infarction; INH, isoniazid; IBD, inflammatory bowel disease; ADAs, antidrug antibodies; URI, upper respiratory tract infection; wk, week; PASI, Psoriasis Area and Severity Index.

Generally, patients who weigh $<90 \mathrm{~kg}$ had better PASI 75 response rates than those weighing $>90 \mathrm{~kg} .{ }^{21}$ The $300 \mathrm{mg}$ dose was rapidly acting and had the highest efficacy, especially in achieving PASI 90/100 among all subgroups including in those over $90 \mathrm{~kg}$, with the greatest maintenance in PASI 75 over time. A recent meta-analysis reviewing clinical trial data found that the $300 \mathrm{mg}$ dose of secukinumab had greater efficacy than the $150 \mathrm{mg}$ dose for both PASI 75 and PASI 90 at week 12, although both were more efficacious than placebo. ${ }^{22}$ Post hoc subgroup analyses suggest that patients with lower body weight and less severe disease may achieve a reasonable response with the $150 \mathrm{mg}$ dose.${ }^{16} \mathrm{~A}$ pen and a prefilled syringe are available, both of which are efficacious and easy to use for self-administration in the JUNCTURE ${ }^{23}$ and FEATURE trials. ${ }^{24}$ A lyophilisate powder requiring reconstitution with sterile water (done by health care professionals) is also available as an injection.

To compare the efficacy of secukinumab with available biologics etanercept and ustekinumab, two Phase III comparator studies were completed. ${ }^{24,25}$ The FIXTURE trial was a 52-week trial that randomly assigned patients to receive either secukinumab $300 \mathrm{mg}, 150 \mathrm{mg}$, placebo, or etanercept per standard dosing with the ability of nonresponders in the placebo group to get rerandomized to the secukinumab dosing groups at week $12 .{ }^{26}$ This study illustrated the superiority of secukinumab $(77.1 \%$ in the $300 \mathrm{mg}$ and $67 \%$ in 150 mg dosing group) to placebo (4.9\%) and etanercept (44\%) in PASI 75 at week 12 as well as the maintenance of PASI 75 from week 12 to week $52(P<0.0001$ for all doses and comparator arm etanercept). ${ }^{26}$ In this study, a higher rate of PASI 90 and 100 was observed with secukinumab $300 \mathrm{mg}$ $\left(54.2 \% \%^{*}+, 24.1 \% *\right)$ and secukinumab $150 \mathrm{mg}(41.9 \% *+$, $\left.14.4 \%^{*}\right)$ compared to etanercept $(20.7 \%, 4.3 \%)$ and placebo $(1.5 \%, 0 \%)$ at week $12\left({ }^{*} P<0.001\right.$ for comparison with etanercept, $+P<0.001$ for comparison with placebo; no PASI 100 responses were observed in the placebo group for comparison). ${ }^{26}$

The CLEAR trial was a 1 -year head-to-head study with 676 subjects who received $300 \mathrm{mg}$ secukinumab or ustekinumab per standard dosing with the primary end point being PASI 90 at week $16 .{ }^{25}$ Previous studies had illustrated a peak PASI at week 16 with PASI 90 being higher with secukinumab ${ }^{25,26}$ compared to PASI 90 responses in Phase III studies for etanercept, ${ }^{27,28}$ adalimumab, ${ }^{29,30}$ and ustekinumab, ${ }^{18,31}$ though these were not head-to-head comparisons. In the CLEAR trial, $79 \%$ of patients in the secukinumab $300 \mathrm{mg}$ group achieved PASI 90 compared to $57.6 \%$ of patients in the ustekinumab group at week 16 $(P<0.0001)$. A secondary outcome of this study was PASI 75 at 4 weeks, which was $50 \%$ in the secukinumab 300 mg group compared to $20.6 \%$ in the ustekinumab group $(P<0.0001)$.

\section{Safety}

For patients with moderate-to-severe psoriasis, secukinumab is generally well-tolerated (Table 1). The most common adverse events of secukinumab, occurring in more than $1 \%$ of patients from pooled analysis of four clinical trials in the first 12 weeks, were nasopharyngitis/ upper respiratory tract infections, diarrhea, rhinitis, oral herpes, pharyngitis, and urticaria. The incidence of nasopharyngitis, diarrhea, and upper respiratory infections were similar between the $300 \mathrm{mg}$ and $150 \mathrm{mg}$ secukinumab groups and the etanercept groups with a higher incidence 
than in placebo groups in the initial 12 weeks. These data when exposure adjusted show an incidence lower or comparable to placebo and etanercept over 52 weeks. There is a boxed warning for infections with the use of secukinumab. ${ }^{16}$

IL-17 plays an important role in mucocutaneous immunity; impaired IL-17 immunity has been associated with Candida infections. ${ }^{32}$ This association is seen with chronic mucocutaneous disease in humans that results in the persistence or recurrence of Candida infections with genetic mutations of IL-17-related genes..$^{33}$ IL-17 is also relevant to neutrophil recruitment, thus its blockade may cause neutropenia. ${ }^{34,35}$ Given these theoretical concerns, adverse events including candidal infections and neutropenia were of special interest. All Candida infections that occurred in the secukinumab study groups were localized, usually to oral or genital candidiasis with severity rated as mild to moderate. ${ }^{23-26}$ These infections were dose dependent among the 300 $\mathrm{mg}$ and $150 \mathrm{mg}$ secukinumab groups. One case of severe gastrointestinal candidiasis was reported in the FIXTURE trial; however, it occurred in the etanercept group. ${ }^{24}$ No cases of chronic mucocutaneous candidiasis were reported in data that was available.

There were 132 patients with either history of tuberculosis or latent tuberculosis enrolled in Phase III trials. There were no cases of reactivation reported; however, a boxed warning recommends screening patients for tuberculosis prior to starting treatment. ${ }^{16}$ There are no published recommendations on when to initiate secukinumab therapy in patients who are found to have latent tuberculosis and who are started on prophylactic therapy. The rates of malignancy were reported to be similar between secukinumab and placebo, with exposure-adjusted indices illustrating a higher rate of malignancies in the placebo group. Caution is advised in patients with hypersensitivity reactions, both the auto-injector pen and prefilled syringe have removable latex caps that should be avoided in those with a latex allergy. ${ }^{16}$ The incidence of antidrug antibodies was $<1 \%$, with very few being neutralizing antibodies. None of these antibodies were associated with loss of clinical efficacy. ${ }^{23-26}$

A final boxed warning regarding the use of secukinumab is in patients with inflammatory bowel disease (IBD). A small ( $\mathrm{N}=59$ ), double-blinded, randomized, multicentere study in Europe was terminated early due to therapeutic inefficacy of intravenous secukinumab in patients with severe Crohn's disease when compared to placebo. ${ }^{36}$ Additionally, more adverse events were reported for patients in the study receiving secukinumab, including reports of worsening
Crohn's flares and infection. ${ }^{36}$ Among the study population in secukinumab Phase III trials, three cases of Crohn's were reported, two flares in patients with existing Crohn's disease, and one new diagnosis of Crohn's disease in a patient with signs and symptoms prior to starting the study trial. There were also two ulcerative colitis flares reported during trial studies and two new diagnoses of ulcerative colitis. ${ }^{16} \mathrm{~A}$ causal relationship between secukinumab and IBD has not been established.

There were a total of six major adverse cardiac events (MACE) in the secukinumab $300 \mathrm{mg}$ group, five MACE events in the secukinumab $150 \mathrm{mg}$ group, 1 MACE event in the placebo and etanercept group in the data available from the FDA..$^{20,24-26}$ These patients all had cardiovascular risk factors, and these events were deemed unsuspected/ unrelated to secukinumab by study investigators. There were seven total deaths reported due to various causes: alcohol intoxication, cerebrovascular accident, intestinal ischemia/hyperkalemia and renal failure, disseminated aspergillosis after liver transplant, myocardial infarction, suicide, and an unknown cause of death in a patient with alcoholic liver disease who died $\sim 3$ months after having been discontinued from the study and receiving his last secukinumab injection..$^{20,24-26}$ None of these deaths were thought to be related to the study medication.

\section{QOL measures}

QOL measures were also evaluated in Phase III clinical trials for secukinumab. The Dermatology Life Quality Index (DLQI) was used to assess impact on QOL, with higher scores signifying greater negative impact on health-related QOL. ${ }^{37}$ The ERASURE trial showed an improvement in DLQI to 0 or 1 as early as week 4 in $46 \%$ of patients in the secukinumab $300 \mathrm{mg}$ group compared to $25 \%$ in the secukinumab $150 \mathrm{mg}$ group and $13.8 \%$ in placebo group $(P<0.01) .{ }^{38}$ Similarly, the FIXTURE trial showed a statistically significant decrease in DLQI by week 12 in a higher proportion of secukinumab patients compared to etanercept and placebo groups $(P<0.001) .{ }^{26}$ The CLEAR trial demonstrated an improved DLQI in a greater proportion of patients at all time points up to week 16 in the secukinumab $300 \mathrm{mg}$ group compared to the ustekinumab group. ${ }^{25}$ In addition, the CLEAR trial also used a subjective symptom assessment to evaluate pain, itching, and scaling on an eleven-point scale with a higher score signifying worsening symptoms. The secukinumab group achieved lower scores in pain, itching, and scaling when compared to the ustekinumab group. ${ }^{25}$ 


\section{Conclusion and place in therapy}

Based on clinical trial data, secukinumab is currently the most efficacious biologic available for the treatment of moderate-to-severe psoriasis with a comparable safety profile to existing biologics. Head-to-head studies illustrated the superiority of secukinumab $300 \mathrm{mg}$ to both etanercept and ustekinumab. Although no head-to-head trial exists comparing the efficacy of adalimumab to secukinumab, a network meta-analysis of randomized controlled data comparing multiple biologics estimates the PASI 75 response rate for adalimumab $40 \mathrm{mg}$ standard dosing protocol to be $\sim 66 \%$, and comparable to the ustekinumab $45 \mathrm{mg}$ dose. ${ }^{39}$ Given the clinical efficacy data presented in this article and the superiority of secukinumab to ustekinumab in the CLEAR trial, it is reasonable to expect that secukinumab is clinically more effective than adalimumab.

Overall, the safety of secukinumab is comparable to other available biologics. Additionally, the analysis of Phase II data has shown very similar safety data among the IL-17 inhibitors secukinumab, brodalumab, and ixekizumab. ${ }^{40}$ The pooled data from the secukinumab trials is close to 4,000 patients. The safety data available will increase with the completion and publications of the Phase III trial data for the other IL-17 inhibitors, brodalumab and ixekizumab. The increased rate of nonserious infections such as nasopharyngitis and upper respiratory infections is also observed with TNF- $\alpha$ blockers. Certain specific safety concerns with the use of secukinumab include possible worsening of IBD such as Crohn's disease, neutropenia, and the occurrence of mild to moderate oral or genital candidiasis.

As more biologics become available, the challenge to practitioners will be how to tailor selection of biologic therapy for patients. Considering certain factors may be helpful in therapeutic decision-making: the presence of psoriatic arthritis, IBD, likelihood of patient adherence, and patient preference regarding more frequent injections, body weight, the formation of antidrug antibodies, or therapeutic failure to a previous biologic..$^{41}$ In patients with psoriatic arthritis or ankylosing spondylitis, secukinumab is an effective treatment choice with recent approval of both indications by the FDA. ${ }^{42,43}$ In patients with IBD, a TNF- $\alpha$ inhibitor such as infliximab and adalimumab is preferred to secukinumab, since these medications provide therapeutic effect and do not carry the risk of potentially worsening IBD. Postmarketing analysis and safety data on other IL-17 inhibitors may be helpful in elucidating the risk of IL-17 inhibition in patients with IBD further.
Considering patient adherence, secukinumab achieves a higher rate of PASI 90 and PASI 100 compared to other available biologics. Thus, secukinumab may be less likely to require combination therapy. Patient preference regarding the frequency of medication administration should also be considered. Initially, the dosing of secukinumab is more frequent during the induction phase with five weekly injections with two syringes followed by monthly injections with two syringes ( $150 \mathrm{mg}$ each). About $150 \mathrm{mg}$ (one syringe) of secukinumab may be considered for use in patients with less severe disease and in those $<90 \mathrm{~kg}$, though this dose is unlikely to be superior to adalimumab or ustekinumab. The $150 \mathrm{mg}$ dose of secukinumab in a metaanalysis evaluating long-term treatment options showed a small significant difference (relative risk [RR] 0.80 with $95 \%$ confidence interval $[\mathrm{CI}] 0.72,0.89)$ relative to etanercept $50 \mathrm{mg}$ dosing per protocol (RR 0.67 with $95 \% \mathrm{CI}$ 0.57, 0.79). ${ }^{44}$ Compared to other available biologics, the maintenance dosing of the secukinumab is less frequent than both etanercept and adalimumab, but more frequent than ustekinumab.

Providers could consider using secukinumab if patients have failed TNF- $\alpha$ agents, because secukinumab targets an alternative immune pathway. The use of secukinumab in the ustekinumab nonresponders is less clear, because its mechanism is more proximal and related to the IL-17 pathway. There are no published studies evaluating the efficacy of secukinumab in patients who have failed prior biologics. In patients who have formed antidrug antibodies to other biologics, treatment with secukinumab could be considered. Secukinumab has a low rate of antidrug antibody formation, none of which were associated with loss of clinical efficacy in clinical trials. ${ }^{20,24-26,45}$

Moderate-to-severe psoriasis has a tremendous negative impact on QOL and work productivity, especially when associated with comorbidities, such as psoriatic arthritis. ${ }^{46}$ Still, undertreatment and dissatisfaction with treatment remain high among patients either due to inefficacy, cost, or difficulty with access. ${ }^{47}$ Finally, the identification of new immune pathways involved in psoriasis and the advent of new biologics such as secukinumab allow providers to achieve higher clearance rates for patients than ever before. Secukinumab specifically achieves quick clearance (PASI 50/75) within 4 weeks and maintenance of PASI 75 in $\sim 70 \%-80 \%$ of patients, PASI 90 in $~ 50 \%-60 \%$, and PASI 100 in $20 \%-40 \%$ of patients based on clinical studies (Table 1). ${ }^{23-26}$ Postmarketing data and further studies using secukinumab in combination therapy, in 
patients who have failed other biologics, and in patients with TNF- $\alpha$-induced psoriasis are of great interest.

\section{Disclosure}

The authors report no conflicts of interest in this work.

\section{References}

1. Rachakonda TD, Schupp CW, Armstrong AW. Psoriasis prevalence among adults in the United States. J Am Acad Dermatol. 2014;70(3): 512-516.

2. Feldman SR, Malakouti M, Koo JY. Social impact of the burden of psoriasis: effects on patients and practice. Dermatol Online J. 2014;20(8). pii: 13030/qt48r4w8h2.

3. Fowler JF, Duh MS, Rovba L, et al. The impact of psoriasis on health care costs and patient work loss. J Am Acad Dermatol. 2008;59(5): $772-780$.

4. Gisondi P, Galvan A, Idolazzi L, Girolomoni G. Management of moderate to severe psoriasis in patients with metabolic comorbidities. Front Med (Lausanne). 2015;2:1.

5. Armstrong AW, Harskamp CT, Armstrong EJ. The association between psoriasis and obesity: a systematic review and meta-analysis of observational studies. Nutr Diabetes. 2012;2:e54.

6. Menter A, Korman NJ, Elmets CA, et al. Guidelines of care for the management of psoriasis and psoriatic arthritis: section 4. Guidelines of care for the management and treatment of psoriasis with traditional systemic agents. J Am Acad Dermatol. 2009;61(3):451-485.

7. Mease P. A short history of biological therapy for psoriatic arthritis. Clin Exp Rheumatol. 2015;33(5 Suppl 93):104-108.

8. Mease PJ, Goffe BS, Metz J, VanderStoep A, Finck B, Burge DJ. Etanercept in the treatment of psoriatic arthritis and psoriasis: a randomised trial. Lancet. 2000;356(9227):385-390.

9. Aggarwal S, Ghilardi N, Xie MH, de Sauvage FJ, Gurney AL. Interleukin-23 promotes a distinct CD4 T cell activation state characterized by the production of interleukin-17. J Biol Chem. 2003;278(3): 1910-1914.

10. Lynde CW, Poulin Y, Vender R, Bourcier M, Khalil S. Interleukin 17A: toward a new understanding of psoriasis pathogenesis. J Am Acad Dermatol. 2014;71(1):141-150.

11. Malakouti M, Brown GE, Wang E, Koo J, Levin EC. The role of IL-17 in psoriasis. J Dermatolog Treat. 2015;26(1): 41-44.

12. Griffiths CE, Reich K, Lebwohl M, et al. Comparison of ixekizumab with etanercept or placebo in moderate-to-severe psoriasis (UNCOVER-2 and UNCOVER-3): results from two phase 3 randomised trials. Lancet. 2015;386(9993):541-551.

13. Lebwohl M, Strober B, Menter A, et al. Phase 3 studies comparing brodalumab with ustekinumab in psoriasis. $N$ Engl J Med. 2015;373(14):1318-1328.

14. Garnock-Jones KP. Secukinumab: a review in moderate to severe plaque psoriasis. Am J Clin Dermatol. 2015;16(4):323-330.

15. Novartis Pharmaceutical Corporation. Advisory Committee Briefing Materials. 2014. Available from http:/www.fda.gov/downloads/ AdvisoryCommittees/CommitteesMeetingMaterials/Drugs/DermatologicandOphthalmicDrugsAdvisoryCommittee/UCM419023.pdf. Accessed October 28, 2015.

16. Novartis Pharmaceuticals Corporation. Cosenytx (Secukinumab) Prescribing Information. East Hanover, NJ: Novartis Pharmaceuticals; 2015.

17. Chioato A, Noseda E, Stevens M, Gaitatzis N, Kleinschmidt A, Picaud H. Treatment with the interleukin-17A-blocking antibody secukinumab does not interfere with the efficacy of influenza and meningococcal vaccinations in healthy subjects: results of an open-label, parallel-group, randomized single-center study. Clin Vaccine Immunol. 2012;19(10): 1597-1602.
18. Papp KA, Langley RG, Lebwohl M, et al. Efficacy and safety of ustekinumab, a human interleukin-12/23 monoclonal antibody, in patients with psoriasis: 52-week results from a randomised, double-blind, placebo-controlled trial (PHOENIX 2). Lancet. 2008;371(9625):1675-1684.

19. Rich P, Sigurgeirsson B, Thaci D, et al. Secukinumab induction and maintenance therapy in moderate-to-severe plaque psoriasis: a randomized, double-blind, placebo-controlled, phase II regimen-finding study. Br J Dermatol. 2013;168(2):402-411.

20. Mrowietz U, Leonardi CL, Girolomoni G, et al. Secukinumab retreatment-as-needed versus fixed-interval maintenance regimen for moderate to severe plaque psoriasis: A randomized, double-blind, noninferiority trial (SCULPTURE). J Am Acad Dermatol. 2015;73(1):27-36.e21.

21. Rich P, Sigurgeirsson B, Thaci DP, et al. Secukinumab induction and maintenance therapy in moderate-to-severe plaque psoriasis: a randomised, double-blind, placebo-controlled, phase II regimen-finding study. Br J Dermatol. 2012.

22. Xiong HZ, Gu JY, He ZG, et al. Efficacy and safety of secukinumab in the treatment of moderate to severe plaque psoriasis: a meta-analysis of randomized controlled trials. Int J Clin Exp Med. 2015;8(3):3156-3172.

23. Paul C, Lacour JP, Tedremets L, et al. Efficacy, safety and usability of secukinumab administration by autoinjector/pen in psoriasis: a randomized, controlled trial (JUNCTURE). J Eur Acad Dermatol Venereol. 2015;29(6):1082-1090.

24. Blauvelt A, Prinz JC, Gottlieb AB, et al. Secukinumab administration by pre-filled syringe: efficacy, safety and usability results from a randomized controlled trial in psoriasis (FEATURE). Br J Dermatol. 2015;172(2):484-493.

25. Thaci D, Blauvelt A, Reich K, et al. Secukinumab is superior to ustekinumab in clearing skin of subjects with moderate to severe plaque psoriasis: CLEAR, a randomized controlled trial. J Am Acad Dermatol. 2015;73(3):400-409.

26. Langley RG, Elewski BE, Lebwohl M, et al. Secukinumab in plaque psoriasis - results of two phase 3 trials. $N$ Engl J Med. 2014;371(4):326-338.

27. Leonardi CL, Powers JL, Matheson RT, et al. Etanercept as monotherapy in patients with psoriasis. N Engl J Med. 2003;349(21):2014-2022.

28. Papp KA, Tyring S, Lahfa M, et al. A global phase III randomized controlled trial of etanercept in psoriasis: safety, efficacy, and effect of dose reduction. Br J Dermatol. 2005;152(6):1304-1312.

29. Menter A, Tyring SK, Gordon K, et al. Adalimumab therapy for moderate to severe psoriasis: a randomized, controlled phase III trial. J Am Acad Dermatol. 2008;58(1):106-115.

30. Saurat JH, Stingl G, Dubertret L, et al. Efficacy and safety results from the randomized controlled comparative study of adalimumab vs. methotrexate vs. placebo in patients with psoriasis (CHAMPION). $\mathrm{Br}$ J Dermatol. 2008;158(3):558-566.

31. Leonardi CL, Kimball AB, Papp KA, et al. Efficacy and safety of ustekinumab, a human interleukin-12/23 monoclonal antibody, in patients with psoriasis: 76-week results from a randomised, double-blind, placebocontrolled trial (PHOENIX 1). Lancet. 2008;371(9625):1665-1674.

32. Conti HR, Gaffen SL. IL-17-mediated immunity to the opportunistic fungal pathogen Candida albicans. J Immunol. 2015;195(3):780-788.

33. Cypowyj S, Picard C, Marodi L, Casanova JL, Puel A. Immunity to infection in IL-17-deficient mice and humans. Eur J Immunol. 2012;42(9):2246-2254.

34. Laan M, Cui ZH, Hoshino H, et al. Neutrophil recruitment by human IL-17 via C-X-C chemokine release in the airways. J Immunol. 1999; 162(4):2347-2352.

35. McCarthy MK, Zhu L, Procario MC, Weinberg JB. IL-17 contributes to neutrophil recruitment but not to control of viral replication during acute mouse adenovirus type 1 respiratory infection. Virology. 2014;456-457:259-267.

36. Hueber W, Sands BE, Lewitzky S, et al. Secukinumab, a human antiIL-17A monoclonal antibody, for moderate to severe Crohn's disease: unexpected results of a randomised, double-blind placebo-controlled trial. Gut. 2012;61(12):1693-1700. 
37. Griffiths CE, Dubertret L, Ellis CN, et al. Ciclosporin in psoriasis clinical practice: an international consensus statement. Br J Dermatol. 2004;150:11-23.

38. Ohtsuki M, Morita A, Abe M, et al. Secukinumab efficacy and safety in Japanese patients with moderate-to-severe plaque psoriasis: subanalysis from ERASURE, a randomized, placebo-controlled, phase 3 study. J Dermatol. 2014;41(12):1039-1046.

39. Signorovitch JE, Betts KA, Yan YS, et al. Comparative efficacy of biological treatments for moderate-to-severe psoriasis: a network metaanalysis adjusting for cross-trial differences in reference arm response. Br J Dermatol. 2015;172(2):504-512.

40. Brown G, Malakouti M, Wang E, Koo JY, Levin E. Anti-IL-17 phase II data for psoriasis: a review. J Dermatolog Treat. 2015;26(1):32-36.

41. Brezinski EA, Armstrong AW. Strategies to maximize treatment success in moderate to severe psoriasis: establishing treatment goals and tailoring of biologic therapies. Semin Cutan Med Surg. 2014;33(2):91-97.

42. Baeten D, Sieper J, Braun J, et al. Secukinumab, an interleukin-17A inhibitor, in ankylosing spondylitis. $N$ Engl J Med. 2015;373(26): 2534-2548.
43. Mease PJ, McInnes IB, Kirkham B, et al. Secukinumab inhibition of interleukin-17A in patients with psoriatic arthritis. NEngl JMed. 2015;373(14): 1329-1339.

44. Nast A, Jacobs A, Rosumeck S, Werner RN. Efficacy and safety of systemic long-term treatments for moderate-to-severe psoriasis: a systematic review and meta-analysis. J Invest Dermatol. 2015;135(11):2641-2648.

45. Karle A, Spindeldreher S, Kolbinger F. Secukinumab, a novel anti-IL17A antibody, shows low immunogenicity potential in human in vitro assays comparable to other marketed biotherapeutics with low clinical immunogenicity. MAbs. 2016;8(3):536-550.

46. Edson-Heredia E, Zhu B, Guo J, Maeda-Chubachi T, Lebwohl M. Disease burden and quality of life in psoriasis patients with and without comorbid psoriatic arthritis: results from National Psoriasis Foundation panel surveys. Cutis. 2015;95(3):173-178.

47. Armstrong AW, Robertson AD, Wu J, Schupp C, Lebwohl MG. Undertreatment, treatment trends, and treatment dissatisfaction among patients with psoriasis and psoriatic arthritis in the United States: findings from the National Psoriasis Foundation surveys, 2003-2011. JAMA Dermatol. 2013;149(10):1180-1185.
Clinical, Cosmetic and Investigational Dermatology

\section{Publish your work in this journal}

Clinical, Cosmetic and Investigational Dermatology is an international, peer-reviewed, open access, online journal that focuses on the latest clinical and experimental research in all aspects of skin disease and cosmetic interventions. This journal is included on PubMed. The manuscript management system is completely online

\section{Dovepress}

and includes a very quick and fair peer-review system, which is all easy to use. Visit http://www.dovepress.com/testimonials.php to read real quotes from published authors

Submit your manuscript here: https:/www.dovepress.com/clinical-cosmetic-and-investigational-dermatology-journal 